\title{
ROOTS OF UNITY AND THE ADAMS-NOVIKOV SPECTRAL SEQUENCE FOR FORMAL $A$-MODULES
}

\author{
KEITH JOHNSON
}

\begin{abstract}
The cohomology of a Hopf algebroid related to the Adams-Novikov spectral sequence for formal $A$-modules is studied in the special case in which $A$ is the ring of integers in the field obtained by adjoining $p$ th roots of unity to $\widehat{Q}_{p}$, the $p$-adic numbers. Information about these cohomology groups is used to give new proofs of results about the $E_{2}$ term of the Adams spectral sequence based on 2-local complex $K$-theory, and about the odd primary Kervaire invariant elements in the usual Adams-Novikov spectral sequence.
\end{abstract}

One of the most powerful tools used in the computation of stable homotopy groups is the Adams-Novikov spectral sequence. The $E_{2}$ term of this spectral sequence is a certain Ext group derived from a universal formal group law. In [R3] the corresponding Ext group for a universal formal $A$-module, for $A$ the ring of algebraic integers in an algebraic number field, $K$, or its $p$ adic completion, was introduced and certain conjectures about these groups were formulated. One of these conjectures (concerning the value of $\mathrm{Ext}^{1, *}$ ) was confirmed in $[\mathrm{J}]$ using a Hopf algebroid (i.e., a generalized Hopf algebra in which the left and right units need not agree), $E_{A} T$, which generalizes the Hopf algebroid $K_{*} K$ of stable cooperations for complex $K$-theory. The present paper is concerned with the cohomology of $E_{A} T$ in the special case of $A=\widehat{\mathbb{Z}}_{p}[\zeta]$ where $\zeta$ is a $p$ th root of unity and $\widehat{\mathbb{Z}}_{p}$ denotes the $p$-adic integers. We will show that in this case $E_{A} T$ is contained in an extension of Hopf algebroids

$$
\widetilde{E_{A} T} \stackrel{j}{\longrightarrow} E_{A} T \stackrel{\rho}{\longrightarrow} \overline{E_{A} T}
$$

and that the cohomology of $\overline{E_{A} T}$ can be completely described. This provides us with information about the cohomology of $E_{A} T$ via the Cartan-Eilenberg spectral sequence associated to this extension.

Two applications of this result are presented. In the case $p=2, E_{A} T$ can be identified with the 2-adic completion of the Hopf algebroid $K_{*} K_{(2)}$ of stable cooperations for 2-primary complex $K$-theory. In this case the cohomology

Received by the editors February 1, 1989.

1980 Mathematics Subject Classification (1985 Revision). Primary 55T25; Secondary 55N22, $14 \mathrm{~L} 05$. 
of $\widetilde{E_{A} T}$ can also be described, so that we can completely describe the CartanEilenberg spectral sequence (there are no nontrivial differentials for dimensional reasons). We therefore obtain a new proof of the results in $[\mathrm{R} 1, \mathrm{~K}]$, computing $H^{*}\left(K_{*} K_{(2)}\right)$.

A second application is the construction of nontrivial elements in the classical Adams-Novikov spectral sequence based on $B P$, the Brown-Peterson spectrum, which is a summand of $M U$, the spectrum representing complex cobordism. In [R3] a map of Hopf algebroids

$$
\Psi: V T=B P_{*} B P \rightarrow V_{A} T
$$

was described (here $V_{A} T$ is the Hopf algebroid generalizing $V T$ to the category of formal $A$-modules, and is constructed using isomorphisms of $A$-typical formal $A$-modules). Composing $\Psi$ with the Conner-Floyd map

$$
\Phi: V_{A} T \rightarrow E_{A} T
$$

constructed in $[\mathrm{J}]$ and the map $\rho$, we have a map

$$
\chi: V T \rightarrow \overline{E_{A} T}
$$

from $V T$ to a Hopf algebroid whose cohomology is known. We thus have a tool for identifying nonzero elements of $H^{* *} V T$, the $E_{2}$ term of the classical Adams-Novikov spectral sequence. We apply this to give a new proof of Theorem 4 of $[R 1]$ concerning the odd primary Kervaire invariant elements.

\section{An extension CONTAining $E_{A} T$}

In this section, we recall the definition and some of the structure of $E_{A} T$. We describe the homogeneous components of $E_{A} T$ and construct two related Hopf algebroids, $\widehat{C}_{n}$ and $\bar{C}_{n}$, with which we construct the extension described in the introduction. We conclude by computing the cohomology of $\bar{C}_{n}$.

The ring $A=\widehat{\mathbb{Z}}_{p}[\zeta]$ is the ring of integers in the field $K=\widehat{\mathbb{Q}}_{p}[\zeta]$, which is an extension of $\widehat{\mathbb{Q}}_{p}$ of degree $p-1 . A$ has a unique prime ideal $(\pi)$ whose generator may be taken to be $\pi=\zeta-1$, and the residue field of $A$, i.e., $A /(\pi)$, is $\mathbb{Z} / p \mathbb{Z} . p$ is totally ramified in $A$, with $(\pi)^{p-1}=(p)$.

Recall from [J] that the Hopf algebroid $\left(E_{A}, E_{A} T\right)$ is defined by

$$
\begin{gathered}
E_{A}=A\left[t, t^{-1}\right], \\
E_{A} T=\left\{f \in K\left[u, u^{-1}, v, v^{-1}\right] \mid f(a t, b t) \in E_{A}, \text { if } a, b \in A, a, b \equiv 1(\pi)\right\}
\end{gathered}
$$

and that $E_{A}, E_{A} T$ are graded with $\operatorname{deg}(t)=\operatorname{deg}(u)=\operatorname{deg}(v)=2(p-1)$. The structure maps for $\left(E_{A}, E_{A} T\right)$ are:

$$
\begin{array}{ll}
\eta_{L}(t)=u, & \eta_{R}(t)=v, \\
\psi(u)=u \otimes 1, & \psi(v)=1 \otimes v, \\
c(u)=v, & c(v)=u, \\
\varepsilon(u)=t, & \varepsilon(v)=t .
\end{array}
$$


If we denote the homogeneous component of $E_{A} T$ of degree $2 \cdot n \cdot(p-1)$ by $\left(E_{A} T\right)_{n}$ then we obtain a Hopf algebroid $\left(A,\left(E_{A} T\right)_{n}\right)$. Let us also define

$$
C_{n}=\left\{f \in K\left[w, w^{-1}\right] \mid f(a) \in A \text { if } a \in A, a \equiv 1(\pi)\right\} .
$$

$\left(A, C_{n}\right)$ can be given the structure of a Hopf algebroid via the maps

$$
\begin{gathered}
\eta_{L}(1)=1, \quad \eta_{R}(1)=w^{n}, \\
\psi(w)=w \otimes w, \quad c(w)=w^{-1}, \quad \varepsilon(w)=1 .
\end{gathered}
$$

We may define a map $C_{n} \rightarrow\left(E_{A} T\right)_{n}$ by $f \mapsto u^{n} \cdot f(v / u)$ and it is straightforward to check that this defines an isomorphism of Hopf algebroids. Thus, in particular we have

$$
H^{s, 2 n \cdot(p-1)}\left(E_{A} T\right) \simeq H^{s}\left(C_{n}\right) .
$$

We will do most of our computations using $C_{n}$ rather than $E_{A} T$, and write $C$ in place of $C_{n}$ if the choice of right unit is not relevant.

Let us also write $B=C \cap K[w]$. We may define a sequence of polynomials in $B$ inductively by

$$
q_{0}=(w-1) / \pi, \quad q_{i+1}=\left(q_{i}^{q}-q_{i}\right) / \pi .
$$

Also, let us denote

$$
q^{I}(w)=q_{0}^{i_{0}} \cdots q_{m}^{i_{m}}
$$

if $I=\left(i_{0}, \ldots, i_{m}\right)$ is a multi-index.

Lemma 1. The polynomials $\left\{q^{I} \mid 0 \leq i_{j}<p, m=0,1,2, \ldots\right\}$ form a basis for $B$ as an A-module.

Proof. This is Proposition 7 of $[\mathrm{J}]$ (note that these polynomials are denoted there by $f_{i}$ ).

Corollary 2. The polynomials $\left\{q_{i} \mid i=0,1, \ldots\right\}$ generate $C$ over $A\left[w, w^{-1}\right]$.

It will be useful for us to have a slightly different generating set for $C$ available in addition to this one. Define inductively

$$
\tilde{q}_{0}=(w-1) / \pi, \quad \tilde{q}_{1}=\left(w^{p}-1\right) / \pi^{p+1}, \quad \tilde{q}_{i+1}=\left(\tilde{q}_{i}^{p}-\tilde{q}_{i}\right) / \pi
$$

and

$$
\tilde{q}^{I}=\tilde{q}_{0}^{i_{0}} \ldots \tilde{q}_{m}^{i_{m}} \quad \text { if } I=\left(i_{0}, \ldots, i_{m}\right) .
$$

Lemma 3. The polynomials $\left\{\tilde{q}^{I} \mid 0 \leq i_{j}<p, m=0,1,2, \ldots\right\}$ form a basis for $B$ as an A-module.

Proof. Part of this lemma is, of course, that $\tilde{q}_{i}(w) \in B$. Since for any $a \in A$, $a^{p}-a \equiv O(\pi)$, it is sufficient for us to show that $\tilde{q}_{1}(w) \in B$. This, however, follows from [J, Lemma 17].

To see that this set forms a basis, note that the $(n+1) \times(n+1)$ matrix that expresses the polynomials $\tilde{q}^{I}$ with $\sum i_{j} p^{j} \leq n$ as a linear combination of the polynomials $q^{I}$ is triangular, with diagonal entries equal to 1 . Thus, it is 
invertible over $A$, and so the polynomials $\tilde{q}^{I} \operatorname{span} B$. They are clearly linearly independent.

Corollary 4. The polynomials $\left\{\tilde{q}_{i} \mid i=0,1, \ldots\right\}$ generate $C$ over $A\left[w, w^{-1}\right]$.

Our interest in this second generating set is motivated by the fact, easily proved by induction, that $\tilde{q}_{i}(w)$ for $i \geq 1$ is a polynomial in $w^{p}$. If we denote $\widetilde{C}=C \cap K\left[w^{p}, w^{-p}\right]$ and $\widetilde{B}=\widetilde{C} \cap K\left[w^{p}\right]$, then we have

Corollary 5. The polynomials $\left\{\tilde{q}^{I} \mid i_{0}=0,0 \leq i_{j}<p, m=1,2, \ldots\right\}$ form $a$ basis for $\widetilde{B}$ as an A-module.

Corollary 6. The polynomials $\left\{\tilde{q}_{i} \mid i=1,2, \ldots\right\}$ generate $\widetilde{C}$ over $A\left[w^{p}, w^{-p}\right]$.

A third algebra related to $C$ and $\widetilde{C}$ is

$$
\widehat{C}=\left\{f \in K\left[x, x^{-1}\right] \mid f(a) \in A \text { if } a \equiv 1\left(\pi^{p+1}\right)\right\} .
$$

We make $\left(A, \widehat{C}_{n}\right)$ into a Hopf algebroid by defining

$$
\begin{gathered}
\eta_{L}(1)=1, \quad \eta_{R}(1)=x^{n}, \\
\psi(x)=x \otimes x, \quad c(x)=x^{-1}, \quad \varepsilon(x)=1 .
\end{gathered}
$$

We also define $\widehat{B}=\widehat{C} \cap K[x]$.

The analogs of the polynomials $q_{i}$ and $\tilde{q}_{i}$ in this case are the polynomials defined by

$$
\hat{q}_{1}=(x-1) / \pi^{p+1}, \quad \hat{q}_{i+1}=\left(\hat{q}_{i}^{p}-\hat{q}_{i}\right) / \pi .
$$

We also use the notation $\hat{q}^{I}=\hat{q}_{1}^{i_{1}} \cdots q_{m}^{i_{m}}$ if $I=\left(i_{1}, \ldots, i_{m}\right)$. The analog of Lemmas 1 and 3 is

Lemma 7. The polynomials $\left\{\hat{q}^{I} \mid 0 \leq i_{j}<p, m=1,2, \ldots\right\}$ form a basis for $\widehat{B}$ as an A-module.

Proof. The map $K[x] \rightarrow K[x]$ defined by $g(x) \mapsto g\left((x-1) / \pi^{p+1}\right)$ maps the algebra of polynomials with the property that $g(a) \in A$ if $a \in A$ isomorphically to $\widehat{B}$. Since it also maps the basis for this former algebra constructed in [J, Proposition 7], onto the set $\left\{\hat{q}^{I}\right\}$, the latter must be a basis for $\widehat{B}$.

Corollary 8. The polynomials $\left\{\hat{q}_{i} \mid i=1,2, \ldots\right\}$ generate $\widehat{C}$ over $A\left[x, x^{-1}\right]$.

The connection between $\widehat{C}$ and the previous two Hopf algebras we have considered is given by

Proposition 9. The map from $\widehat{C}$ to $C$ that sends $x$ to $w^{p}$ is an injection of Hopf algebroids whose image is $\widetilde{C}$.

Proof. Since this map sends $\hat{q}_{i}$ to $\tilde{q}_{i}$, the result is clear.

We next describe the Hopf algebroid $\left(\bar{E}_{A}, \bar{E}_{A} \bar{T}\right)$, or rather we describe its homogeneous, degree $n \cdot 2 \cdot(p-1)$ component, $\bar{C}_{n}$. Let $\bar{C}_{n}$ denote the dual of the group algebra of the cyclic group of order $p$ :

$$
\bar{C}_{n}=A[\mathbb{Z} / p \mathbb{Z}]^{*}=\operatorname{Hom}_{A}(A[\mathbb{Z} / p \mathbb{Z}], A) .
$$


The structure maps for $\bar{C}_{n}$ are, using $\delta$ to denote a generator for $\mathbb{Z} / p \mathbb{Z}$,

$$
\begin{gathered}
\psi(f)\left(\delta^{i} \otimes \delta^{j}\right)=f\left(\delta^{i+j}\right), \\
\eta_{L}(1)\left(\delta^{i}\right)=1, \quad \eta_{R}(1)\left(\delta^{i}\right)=\zeta^{n \cdot i}, \\
c(f)\left(\delta^{i}\right)=f\left(\delta^{-i}\right), \quad \varepsilon(f)=f(1) .
\end{gathered}
$$

Let us also define a map of Hopf algebroids $\rho:\left(A, C_{n}\right) \rightarrow\left(A, \bar{C}_{n}\right)$ by $\rho(f)\left(\delta^{i}\right)$ $=f\left(\zeta^{i}\right)$.

The critical fact about $\rho$ is

Lemma 10. $\rho$ is a normal map of Hopf algebroids.

Proof. It is straightforward that $\rho$ preserves the Hopf algebroid structure maps and so defines a map of Hopf algebroids; the question is whether it is normal. Referring to [R4, A1.1.10] we must verify that

$$
C_{n} \square \square_{\bar{C}_{n}^{\prime}} A=A \square_{\bar{C}_{n}^{\prime}} C_{n}
$$

where $\square$ denotes the cotensor product and, for $(A, \Gamma)$ a Hopf algebroid, $\Gamma^{\prime}$ is the associated Hopf algebra, defined by

$$
\Gamma^{\prime}=\Gamma /\left(\eta_{R}(a)-\eta_{L}(a) \mid a \in A\right) .
$$

In the case $\Gamma=\bar{C}_{n}$, this becomes

$$
\bar{C}_{n}^{\prime}= \begin{cases}\bar{C}_{n} & \text { if } p \mid n, \\ A & \text { if }(p, n)=1 .\end{cases}
$$

To see this, note that if $p \mid n$, then

$$
\begin{aligned}
\left(\eta_{R}(a)-\eta_{L}(a)\right)\left(\delta^{i}\right) & =a \cdot\left(\eta_{R}(1)-\eta_{L}(1)\right)\left(\delta^{i}\right) \\
& =a \cdot\left(\zeta^{n^{\circ} i}-1\right)=a \cdot(1-1)=0
\end{aligned}
$$

while if $(n, p)=1$, then the ideal generated by $\eta_{R}(a)-\eta_{L}(a)$ is

$$
I=\left\{\phi \in \bar{C}_{n} \mid \phi(1)=0\right\} .
$$

Thus, the map $\bar{C}_{n}^{\prime}=\bar{C}_{n} / I \rightarrow A$ that sends $\phi$ to $\phi(1)$ is an isomorphism.

Since $C_{n} \square_{A} A=A \square_{A} C_{n}=C_{n}$, we may assume that $p \mid n$. The cotensor product $C_{n} \square_{\bar{C}_{n}} A$ is defined to be the kernel of the map

$$
C_{n} \simeq C_{n} \otimes_{A} A \rightarrow C_{n} \otimes_{A} \bar{C}_{n} \otimes_{A} A
$$

which sends $f$ to $(1 \otimes \rho)(\psi f) \otimes 1-f \otimes \eta_{L}(1) \otimes 1$. This kernel consists of those elements $f \in \bar{C}_{n}$ for which

$$
(1 \otimes \rho)(f(w \otimes w))=f(w) \otimes 1
$$

in $C_{n} \otimes_{A} \bar{C}_{n}$. These are precisely those elements $f \in \bar{C}_{n}$ of the form $f(w)=$ $g\left(w^{p}\right)$. Similarly, $A \square{\overline{C_{n}}}_{n} C_{n}$ consists of those $f \in C_{n}$ for which

$$
(\rho \otimes 1)(f(w \otimes w))=\eta_{R}(1) \otimes f(w)
$$


in $\bar{C}_{n} \otimes C_{n}$. Since $\eta_{R}(1)=1$ in $\bar{C}_{n}$ when $p \mid n$, we see that this also consists of those $f \in \bar{C}_{n}$ of the form $f(w)=g\left(w^{p}\right)$.

If we define the sub-Hopf algebroid $\left(\tilde{\tilde{A}}, \tilde{\tilde{C}}_{n}\right)$ of $\left(A, C_{n}\right)$ by

$$
\tilde{\tilde{A}}=A \square_{\bar{C}_{n}^{\prime}} A, \quad \tilde{\tilde{C}}_{n}=A \square \square_{\bar{C}_{n}} C_{n} \square_{\bar{C}_{n}} A,
$$

then, following [R4, A1.1.15], we have

Corollary 11. $\left(\tilde{\tilde{A}}, \tilde{\tilde{C}}_{n}\right) \stackrel{i}{\longrightarrow}\left(A, C_{n}\right) \stackrel{\rho}{\longrightarrow}\left(A, \bar{C}_{n}\right)$ is an extension of Hopf algebroids. (The fact that $i$ is an inclusion is [R4, A1.1.14].)

For this to be useful we must describe $\left(\tilde{\tilde{A}}, \tilde{\tilde{C}}_{n}\right)$. As noted in the proof of [R4, A 1.1.14], we have

$$
\begin{gathered}
\tilde{\tilde{A}}=\left\{a \in A \mid \eta_{L}(a)=\eta_{R}(a) \text { in } \bar{C}_{n}\right\}, \\
\tilde{\tilde{C}}_{n}=\left\{f \in C_{n} \mid(\rho \otimes 1 \otimes \rho) \psi^{2} f=\eta_{L}(1) \otimes f \otimes \eta_{R}(1)\right\} .
\end{gathered}
$$

and so

$$
\left(\tilde{\tilde{A}}, \tilde{\tilde{C}}_{n}\right)= \begin{cases}0 & \text { if }(n, p)=1, \\ \left(A, \widetilde{C}_{n}\right) \simeq\left(A, \widehat{C}_{n / p}\right) & \text { if } n \mid p .\end{cases}
$$

The applications we have in mind for this extension involve the cohomology of $C_{n}$, which we approach via that of $\widetilde{C}_{n}$ and $\bar{C}_{n}$. We conclude this section, therefore, by recalling the cohomology of $\bar{C}_{n}$. Let us define two homomorphisms $S, T: \bar{C}_{n} \rightarrow \bar{C}_{n}$ by

$$
S(f)(x)=f(\delta x)-f(x) \quad \text { and } \quad T(f)(x)=\sum_{i=0}^{p-1} f\left(\delta^{i} x\right) .
$$

A straightforward computation yields

Lemma 12. $0 \rightarrow A \stackrel{\eta_{L}}{\longrightarrow} \bar{C}_{n} \stackrel{S}{\longrightarrow} \bar{C}_{n} \stackrel{T}{\longrightarrow} \bar{C}_{n} \stackrel{S}{\longrightarrow} \cdots$ is an injective resolution of $A$ considered as a left $C_{n}$ comodule.

Corollary 13. The cohomology of $\bar{C}_{n}$ is given by

$$
H^{s}\left(\bar{C}_{n}\right)= \begin{cases}A / \pi A, & s \text { odd }, \\ 0, & \text { s even },\end{cases}
$$

if $(n, p)=1$, and by

$$
H^{s}\left(\bar{C}_{n}\right)= \begin{cases}A, & s=0, \\ A / p A, & s>0, \text { s even }, \\ 0, & s \text { odd },\end{cases}
$$

if $p \mid n$.

Proof. Applying the functor $A \square_{C_{n}}$ ( ) to the resolution of $A$ and using the identification $A \square_{C_{n}} C_{n}=A$ gives the complexes

$$
A \stackrel{\zeta^{n}-1}{\longrightarrow} A \stackrel{0}{\longrightarrow} A \stackrel{\zeta^{n}-1}{\longrightarrow} A \stackrel{0}{\longrightarrow} \cdots
$$


if $(n, p)=1$, and

$$
A \stackrel{0}{\longrightarrow} A \stackrel{p}{\longrightarrow} A \stackrel{0}{\longrightarrow} A \stackrel{p}{\longrightarrow} A \longrightarrow \cdots
$$

if $p \mid n$.

\section{Applications}

2.1. The cohomology of $K_{*} K_{(2)}$. If the prime $p$ is chosen to be 2 , then $A=\widehat{\mathbb{Z}}_{2}$ and $C_{n}$ can be described as

$$
C_{n}=\left\{f \in \widehat{\mathbb{Q}}_{2}\left[w, w^{-1}\right] \mid f(a) \in \widehat{\mathbb{Z}}_{2} \text { if } a \equiv 1(2)\right\} .
$$

The description of $K_{*} K$ given in [AHS]

$$
\begin{aligned}
& K_{*} K=\left\{f \in \mathbb{Q}\left[u, u^{-1}, v, v^{-1}\right] \mid f(a t, b t) \in \mathbb{Z}\left[t, t^{-1}, 1 / a, 1 / b\right]\right. \\
&\text { if } a, b \in \mathbb{Z}, a, b \neq 0\}
\end{aligned}
$$

shows that $C_{n}$ can be identified with $\left(K_{*} K_{(2)}\right)_{n}$ so that the $E_{2}$ term of the Adams spectral sequence based on 2-local complex $K$-theory has as its completion

$$
E_{2}^{*, n}=H^{*}\left(C_{n}\right)=\operatorname{Ext}_{C_{n}}^{*}\left(\widehat{\mathbb{Z}}_{2}, \widehat{\mathbb{Z}}_{2}\right) .
$$

The Cartan-Eilenberg spectral sequence, [R4, A1.3.14], allows us to describe these groups in terms of the cohomology of $\widetilde{C}_{n}$ and $\bar{C}_{n}$ :

Proposition 14. There is a spectral sequence converging to $\operatorname{Ext}_{C_{n}}^{*}\left(\widehat{\mathbb{Z}}_{2}, \widehat{\mathbb{Z}}_{2}\right)$ whose $E_{2}$ term is $E_{2}^{s, t}=\operatorname{Ext}_{\widetilde{C}_{n}}^{s}\left(\widehat{\mathbb{Z}}_{2}, \operatorname{Ext}_{\bar{C}_{n}}^{t}\left(\widehat{\mathbb{Z}}_{2}, \widehat{\mathbb{Z}}_{2}\right)\right)$.

Since $\operatorname{Ext}_{\bar{C}_{n}}^{*}\left(\widehat{\mathbb{Z}}_{2}, \widehat{\mathbb{Z}}_{2}\right)$ is described at the end of $\S 1$, we turn to describing $\operatorname{Ext}_{\widetilde{C}_{n}}^{*}\left(\widehat{\mathbb{Z}}_{2},\right)$. The key to this description is the following injective resolution, which is the analog at the prime 2 of a resolution constructed for odd primes in $[\mathrm{B}, \S 7]$.

Lemma 15. The sequence

$$
0 \rightarrow \widehat{\mathbb{Z}}_{2} \stackrel{p_{0}}{\longrightarrow} \widehat{C}_{n} \stackrel{p_{1}}{\longrightarrow} \widehat{C}_{n} \stackrel{p_{2}}{\longrightarrow} \widehat{\mathbb{Q}}_{2} \rightarrow 0
$$

defined by $p_{1}(f)=f(9 w)-f(w)$ and $p_{2}\left(\sum a_{i} w^{i}\right)=a_{0}$ is an injective resolution of $\widehat{\mathbb{Z}}_{2}$.

(The left $\widehat{C}_{n}$ comodule structure of $\widehat{\mathbb{Z}}_{2}$ and $\widehat{\mathbb{Q}}_{2}$ is that defined by $\eta_{L}$.)

(The factor $9=2^{3}+1$ occurs here because it is a generator of

$$
\left(1+2^{3} \widehat{\mathbb{Z}}_{2}\right) /\left(1+2^{n} \widehat{\mathbb{Z}}_{2}\right)
$$

for $n \geq 4$.)

Proof. If $p_{1}\left(\sum a_{i} w^{i}\right)=\sum_{i} a_{i}\left(9^{i}-1\right) \cdot w^{i}=0$ then $a_{i}=0$ for $i \neq 0$ and the integrality condition for $\widehat{C}_{n}$ shows that $a_{0} \in \widehat{\mathbb{Z}}_{2}$. Thus, $\operatorname{ker}\left(p_{1}\right)=\operatorname{Im}\left(p_{0}\right)$. 
The fact that the polynomials $\left(w^{2^{k}}-1\right) / 2^{k+3}$ are in $\widehat{C}_{n}$ shows that $p_{2}$ is surjective.

It remains to verify that $\operatorname{ker}\left(p_{2}\right)=\operatorname{Im}\left(p_{1}\right)$. Suppose that

$$
f=\sum_{i \neq 0} a_{i} w^{i} \in \operatorname{ker}\left(p_{2}\right) .
$$

For any $a \in \widehat{\mathbb{Q}}_{2}$, the polynomial

$$
g(w)=a+\sum_{i \neq 0} \frac{a_{i} w^{i}}{9^{i}-1}
$$

is mapped to $f$ by $p_{1}$. The question is whether $a$ can be chosen so that $g \in$ $\widehat{C}_{n}$. Choose $a$ so that $g(1)=0$. Since $p_{1}(g)=g(9 w)-g(w) \in \widehat{C}_{n}$ it follows by induction on $k$ that $g\left(9^{k}\right) \in A$ for any $k$, and this is enough to imply $g \in$ $\widehat{C}_{n}$. To see this, first note that there exists $m$ such that $2^{m} \cdot g \in \widehat{\mathbb{Z}}_{2}\left[w, w^{-1}\right]$, and such that if $a, b \in \widehat{\mathbb{Z}}_{2}$, then $g(b) \in \widehat{\mathbb{Z}}_{2}$. However, $\left(1+2^{3} \widehat{\mathbb{Z}}_{2}\right) /\left(1+2^{m} \widehat{\mathbb{Z}}_{2}\right)$ is cyclic, generated by 9 . Thus, if $a \in 1+2^{3} \widehat{\mathbb{Z}}_{2}$, then $a \equiv 9^{k} \bmod 2^{m}$ for some $k$ and so $g(a) \in \widehat{\mathbb{Z}}_{2}$.

\section{Corollary 16.}

(a)

$$
\operatorname{Ext}_{\widehat{C}_{n}}^{s}\left(\widehat{\mathbb{Z}}_{2}, \widehat{\mathbb{Z}}_{2}\right)= \begin{cases}\widehat{\mathbb{Z}}_{2}, & s=0, \\ \widehat{\mathbb{Q}}_{2} / \widehat{\mathbb{Z}}_{2}, & s=2, \\ 0, & \text { otherwise; }\end{cases}
$$

(b) for $n \neq 0$

$$
\operatorname{Ext}_{\widehat{C}_{n}}^{s}\left(\widehat{\mathbb{Z}}_{2}, \widehat{\mathbb{Z}}_{2}\right)= \begin{cases}\mathbb{Z} / 2^{d(n)} \mathbb{Z}, & s=1, \\ 0, & \text { otherwise }\end{cases}
$$

(c)

$$
\operatorname{Ext}_{\widehat{C}_{n}}^{s}\left(\widehat{\mathbb{Z}}_{2}, \mathbb{Z} / 2 \mathbb{Z}\right)= \begin{cases}\mathbb{Z} / 2 \mathbb{Z}, & s=0,1, \\ 0, & \text { otherwise. }\end{cases}
$$

Here $d(n)$ is the largest integer such that $2^{d(n)}$ divides $2^{3} \cdot n$. Proof. $\operatorname{Ext}_{\widehat{C}_{n}}\left(\widehat{\mathbb{Z}}_{2}, \widehat{\mathbb{Z}}_{2}\right)$ is the cohomology of the complex

$$
\widehat{\mathbb{Z}}_{2} \square \widehat{C}_{n} \widehat{C}_{n} \rightarrow \widehat{\mathbb{Z}}_{2} \square_{\widehat{C}_{n}} \widehat{C}_{n} \rightarrow \widehat{\mathbb{Z}}_{2} \square_{\widehat{C}_{n}} \widehat{\mathbb{Q}}_{2} \rightarrow 0
$$

If $n=0$ this complex is

$$
\widehat{\mathbb{Z}}_{2} \stackrel{0}{\longrightarrow} \widehat{\mathbb{Z}}_{2} \stackrel{1}{\longrightarrow} \widehat{\mathbb{Q}}_{2} \rightarrow 0
$$

and, if $n \neq 0$

$$
\widehat{\mathbb{Z}}_{2} \stackrel{9^{n}-1}{\longrightarrow} \widehat{\mathbb{Z}}_{2} \longrightarrow 0 \longrightarrow 0
$$


These account for (a) and (b), since the highest power of 2 dividing $9^{n}-1$ is $2^{d(n)}$. For (c), we are interested in the cohomology of

$$
\widehat{\mathbb{Z}}_{2} \square_{\widehat{C}_{n}} \widehat{C}_{n} \otimes_{A} \mathbb{Z} / 2 \mathbb{Z} \rightarrow \widehat{\mathbb{Z}}_{2} \square_{\widehat{C}_{n}} \widehat{C}_{n} \otimes_{A} \widehat{\mathbb{Z}} / 2 \mathbb{Z} \rightarrow \widehat{\mathbb{Z}}_{2} \square_{\widehat{C}_{n}} \otimes_{A} \mathbb{Z} / 2 \mathbb{Z} \rightarrow 0 .
$$

This complex is, for any $n$,

$$
\mathbb{Z} / 2 \mathbb{Z} \stackrel{0}{\rightarrow} \mathbb{Z} / 2 \mathbb{Z} \rightarrow 0 \text {. }
$$

Combining these results with Proposition 14 and Corollary 13, we obtain Corollary 17. The $E_{2}$ term of the spectral sequence of Proposition 14 is

$$
E_{2}^{s, t}= \begin{cases}\widehat{\mathbb{Z}}_{2}, & \text { if }(s, t)=(0,0), n=0, \\ \widehat{\mathbb{Q}}_{2} / \widehat{\mathbb{Z}}_{2}, & \text { if }(s, t)=(2,0), n=0, \\ \mathbb{Z} / 2^{d(m)} \mathbb{Z}, & \text { if }(s, t)=(1,0), n=2 m=0, \\ \mathbb{Z} / 2 \mathbb{Z}, & \text { if }(s, t)=\left(0,2 t^{\prime}\right), n=2 m, \text { or }\left(1,2 t^{\prime}\right), \\ 0, & \text { otherwise. }\end{cases}
$$

Corollary 18.

$\mathrm{Ext}_{K_{*} K_{(2)}, t}\left(\pi_{*} K, \pi_{*} K\right)= \begin{cases}\mathbb{Z}_{(2)}, & \text { if }(s, t)=(0,0), \\ \mathbb{Z} / 2^{\infty} \mathbb{Z} \oplus \mathbb{Z} / 2 \mathbb{Z}, & \text { if }(s, t)=(2,0), \\ \mathbb{Z} / 2^{d(m)} \mathbb{Z}, & \text { if }(s, t)=(1,2 m) \neq(1,0), \\ \mathbb{Z} / 2 \mathbb{Z}, & \text { if }(s, t)=\left(s, 2 t^{\prime}\right) \neq(2,0), s \geq 2, \\ 0, & \text { otherwise. }\end{cases}$

2.2. The odd primary Kervaire invariant elements. The Hopf algebroid $\left(V_{A}, V_{A} T\right)$ is constructed using isomorphisms of $A$-typical formal $A$-modules. If $A=\mathbb{Z}_{(p)}$, then one obtains $(V, V T)=\left(B P_{*}, B P_{*} B P\right)$, the Hopf algebroid of BrownPeterson homology. If $A$ is a $\mathbb{Z}_{(p)}$ algebra as in the case $A=\widehat{\mathbb{Z}}_{p}[\zeta]$ with which we are concerned, then a formal $A$-module is also a formal $\mathbb{Z}_{(p)}$ module. Thus, we obtain, as in [R3, 3.11], as map of Hopf algebroids

$$
\Psi:(V, V T) \rightarrow\left(V_{A}, V_{A} T\right) .
$$

Composing this with the generalized Conner-Floyd map

$$
\Phi:\left(V_{A}, V_{A} T\right) \rightarrow\left(E_{A}, E_{A} T\right)
$$

of $[\mathrm{J}]$ and with $\rho:\left(E_{A}, E_{A} T\right) \rightarrow\left(E_{A}, \overline{E_{A} T}\right)$ we obtain a map

$$
\chi:(V, V T) \rightarrow\left(E_{A}, \overline{E_{A} T}\right)
$$

and so a map in cohomology

$$
\chi^{*}: H^{*} V T \rightarrow H^{*}\left(E_{A} T\right) .
$$

We will show that a family of interesting elements in $H^{*} V T$, the odd primary Kervaire invariant elements, have nonzero image under this map. 
Recall that $(V, V T)$ has the description

$$
V=\mathbb{Z}_{(p)}\left[v_{1}, v_{2}, \ldots\right], \quad V T=V\left[t_{1}, t_{2}, \ldots\right],
$$

and that $V, V T$ are graded with $\operatorname{deg}\left(v_{i}\right)=\operatorname{deg}\left(t_{i}\right)=2\left(p^{i}-1\right)$. The elements $h_{0}, b_{i} \in H^{1,2(p-1)}(V T), H^{2,2(p-1) p^{i+1}}(V T)$, respectively, are represented in the cobar complex of $V T$ by $h_{0}=\left[t_{1}\right]$ and

$$
b_{i}=\frac{1}{p} \sum_{j=1}^{p^{i+1}-1}\left(p_{j}^{i+1}\right)\left[t_{1}^{j} \otimes t_{1}^{p^{i+1}-j}\right] .
$$

Our result is

Proposition 19. All monomials in $h_{0}, b_{i}, i=0,1,2, \ldots$, have nonzero image in $H^{*}\left(E_{A} T\right)$ under $\chi^{*}$.

Proof. It is straightforward to describe the map of cobar complexes induced by $\chi$. We also need, however, a method of identifying cohomologically nontrivial elements in the cobar complex of $E_{A} T$ or $\bar{C}_{n}$. For this we define a chain map from the cobar complex of $\bar{C}_{n}$ to the complex described in $\S 1$, Lemma 12.

Recall from [R4, A1.2.11] that the cobar resolution of $A$ as a $\bar{C}_{n}$ comodule has as its $s$ th term $\bar{C}_{n} \otimes(\operatorname{ker}(\varepsilon))^{\otimes s}$ and that the differential is given by

$$
d\left(\gamma_{0} \otimes \cdots \otimes \gamma_{s}\right)=\sum_{i=0}^{s}(-1)^{s} \gamma_{0} \otimes \cdots \otimes \psi\left(\gamma_{i}\right) \otimes \cdots \otimes \gamma_{s}+(-1)^{s+1} \gamma_{0} \otimes \cdots \otimes \gamma_{s} .
$$

If we identify elements of

$$
\bar{C}_{n} \otimes(\operatorname{ker}(\varepsilon))^{\otimes s} \subseteq \bar{C}_{n}^{\otimes s+1}=\operatorname{Hom}_{A}(A[\mathbb{Z} / p \mathbb{Z}], A)^{\otimes s+1}
$$

with multilinear maps from $A[\mathbb{Z} / p \mathbb{Z}]^{s+1}$ to $A$, then the differential becomes

$$
\begin{aligned}
d f\left(w_{0}, \ldots, w_{s+1}\right)= & \sum_{i=0}^{s}(-1)^{i} f\left(w_{0}, \ldots, w_{i} \cdot w_{i+1}, \ldots, w_{s+1}\right) \\
& +(-1)^{s+1} f\left(w_{0}, \ldots, w_{s}\right) .
\end{aligned}
$$

Using this identification we define a chain map, $R$, from the cobar resolution of $A$ over $\bar{C}_{n}$ to the resolution described in Lemma 12 .

$$
R(f)(w)= \begin{cases}f(w, \zeta) \quad \text { if } s=1, & s \text { odd } \\ \sum_{i_{1}, \ldots, i_{s-1 / 2}=1}^{p-1} f\left(w, \zeta^{i_{1}}, \zeta, \zeta^{i_{2}}, \ldots, \zeta\right), & \\ \sum_{i_{1}, \ldots, i_{s / 2}=1}^{p-1} f\left(w, \zeta, \zeta^{i_{1}}, \zeta, \zeta^{i_{2}}, \ldots, \zeta^{i_{s / 2}}\right), & s \text { even. }\end{cases}
$$

Applying $A \square_{C_{n}}$ ( ) we obtain a map from the cobar complex of $\bar{C}_{n}$ to the complex of Corollary 13. We denote this map by $R$ as well. It is given by

$$
R(f)= \begin{cases}f(\zeta), & s=1, \\ \sum f\left(\zeta^{i_{1}}, \zeta, \zeta^{i_{2}}, \ldots, \zeta\right), & s \text { odd } \\ \sum f\left(\zeta^{i_{1}}, \zeta, \zeta^{i_{2}}, \ldots, \zeta^{i_{s / 2}}\right), & s \text { even } .\end{cases}
$$


Under the composition $\Phi \circ \Psi$, the elements $h_{0}$ and $b_{i}$ are mapped to $(w-1) / \pi$ and

$$
\frac{1}{p} \sum_{j=1}^{p^{i+1}-1}\left(\begin{array}{c}
p^{i+1} \\
j
\end{array}\right)\left(\frac{w-1}{\pi}\right)^{j} \otimes\left(\frac{w-1}{p}\right)^{p^{i+1}-j}
$$

in the cobar complex of $C_{n}$. Under the composition $R \circ \rho$, these are mapped to 1 and

$$
\frac{1}{p} \sum_{j=1}^{p-1}\left(\left(\frac{\zeta^{j}-1}{\pi}+1\right)^{p^{i+1}}-\left(\frac{\zeta^{j}-1}{\pi}\right)^{p^{i+1}}-1\right)
$$

respectively. We denote the latter element of $A$ by $k_{i}$. This series of maps will send the monomial $h_{0}^{\varepsilon} b_{1}^{i_{1}} \cdots b_{m}^{i_{m}}$ to $k_{1}^{i_{1}} \cdots k_{m}^{i_{m}}$. Showing that $k_{i} \not \equiv 0 \bmod \pi$ will, therefore, complete the proof of Proposition 19.

$$
\begin{aligned}
\frac{1}{p} \sum_{j=1}^{p-1} & \left(\left(\frac{\zeta^{i}-1}{\pi}+1\right)^{p^{i+1}}-\left(\frac{\zeta^{i}-1}{\pi}\right)^{p^{i+1}}-1\right) \\
& =\frac{1}{p} \sum_{j=1}^{p-1} \sum_{k=1}^{p-1^{i+1}}\left(\begin{array}{c}
p^{i+1} \\
k
\end{array}\right)\left(\frac{\zeta^{i}-1}{\pi}\right)^{k} \\
& \equiv \frac{1}{p} \sum_{j=1}^{p-1} \sum_{k=1}^{p-1}\left(\begin{array}{l}
p^{m+1} \\
k \cdot p^{m}
\end{array}\right)\left(\frac{\gamma^{j-1}}{\pi}\right)^{k \cdot p^{m}} \bmod \pi \\
& \equiv \frac{1}{p} \sum_{j=1}^{p-1} \sum_{k=1}^{p-1}\left(\begin{array}{l}
p \\
k
\end{array}\right)\left(\frac{\gamma^{j-1}}{\pi}\right)^{k} \bmod \pi \\
& \equiv \frac{1}{p} \sum_{j=1}^{p-1} \sum_{k=1}^{p-1}\left(\begin{array}{l}
p \\
k
\end{array}\right) j^{k} \bmod \pi \\
& =\frac{1}{p} \sum_{k=1}^{p-1}\left(\begin{array}{l}
p \\
k
\end{array}\right) \sum_{j=1}^{p-1} j^{k} \equiv \frac{-1}{p}\left(\begin{array}{c}
p \\
p-1
\end{array}\right) \bmod \pi \\
& \equiv-1 \bmod \pi .
\end{aligned}
$$

In these congruences, we have used the fact that $\left(\begin{array}{c}p_{k}^{i+1} \\ k\end{array}\right)$ is divisible by $p^{2}$ unless $k$ is divisible by $p^{i}$, that $\left(\begin{array}{c}p^{i+1} \\ k p^{i}\end{array}\right) \equiv\left(\begin{array}{l}p \\ k\end{array}\right) \bmod p$, that since $\zeta=\pi+1$, $\left(\left(\zeta^{i}-1\right) / \pi\right) \equiv i \bmod \pi$, and, finally, that $\sum_{j=1}^{p-1} j^{k} \equiv 0 \bmod p$ if $k<p-1$ and that $\sum_{j=1}^{p-1} j^{p-1} \equiv-1 \bmod p$. 


\section{REFERENCES}

[AHS] J. F. Adams, A. S. Harris, and R. M. Switzer, Hopf algebras of cooperations for real and complex K-theory, Proc. London Math. Soc. (3) 23 (1971), 385-408.

[B] A. K. Bousfield, On the homotopy theory of $K$-local spectra at an odd prime, Amer. J. Math. 107 (1985), 895-932.

[J] K. Johnson, The Conner-Floyd map for formal A-modules, Trans. Amer. Math. Soc. 302 (1987), 319-332.

[K] W. Kreuger, The 2-primary $K$-theory Adams spectral sequence, J. Pure Appl. Algebra 36 (1985), 143-158.

[R1] D. C. Ravenel, The non-existence of odd primary Arf invariant elements in stable homotopy theory, Proc. Cambridge Philos. Soc. 83 (1978), 429-443.

[R2] _ Localization with respect to certain periodic homology theories, Amer. J. Math. 106 (1984), 351-414.

[R3] _ Formal A-modules and the Adams Novikov spectral sequence, J. Pure Appl. Algebra 32 (1984), 327-345.

[R4] C_ Complex cobordism and stable homotopy groups of spheres, Academic Press, New York, 1986.

Department of Mathematics, Statistics and Computing Science, Dalhousie University, Halifax, Nova Scotia, Canada 